\title{
Traverse Down
}

National Cancer Institute

\section{Source}

National Cancer Institute. Traverse Down. NCI Thesaurus. Code C44243.

Specifies the default behavior of a tree search or traversal. 